This document is the accepted manuscript version of the following article:

Asakura, K., Gaffney, K. J., Mi1ne, C., \& Yabashi, M. (2020). XFELs: cutting edge X-ray 1ight

for chemical and material sciences. Physical Chemistry Chemical Physics, 22(5), $2612-2614$.

https://doi .org/10.1039/c9cp90304f

\title{
XFELs: cutting edge X-ray light for chemical and material sciences
}

\author{
Kiyotaka Asakura, (D) *a Kelly J. Gaffney, (D) ${ }^{* b}$ Christopher Milne (D) ${ }^{\mathrm{c}}$ and \\ Makina Yabashi iD *d
}

DOI: $10.1039 / c 9 c p 90304 f$

rsc.li/pccp

Optical lasers (light amplification by stimulated emission of radiation) have been central to chemical and molecular research since their invention in the early 1960s. Optical laser technology has spurred many great innovations in the spectroscopy of chemical species, such as ultra-fast pump-probe spectroscopy, resonant Raman spectroscopy, sum frequency generation spectroscopy, multiphoton absorption and ionization, laser induced fluorescence, cavity ring down spectroscopy, and confocal laser microscopy, among others. ${ }^{1,2} \mathrm{X}$-rays have been equally impactful, providing a critical approach to characterizing the electronic and nuclear structure of molecules and materials with atomic resolution and specificity. Until recently, however, the dominant sources of X-rays have lacked both the temporal and spatial coherence characteristics of a laser. The past decade has seen a transformation in X-ray laser capabilities and this themed issue highlights research advances in chemical and materials science enabled by X-ray laser sources.

There are two ways to extend lasers into the X-ray wavelength regime. One approach

\footnotetext{
${ }^{a}$ Institute for Catalysis, Hokkaido University, Sapporo 001-0021, Hokkaido, Japan. E-mail:askr@cat.hokudai.ac.jp

${ }^{b}$ PULSE Institute, SLAC National Accelerator Laboratory, Stanford University, Stanford, 94305, California, USA. E-mail: kgaffney@slac.stanford.edu ${ }^{c}$ Paul Scherrer Institute, 5232 Villigen-PSI, Switzerland. E-mail: chris.milne@psi.ch

${ }^{d}$ RIKEN SPring-8 Center, Kouto 1-1-1 Sayo, Hyogo 679-5148, Japan.E-mail: yabashi@spring8.or.jp
}

is to use high harmonic generation (HHG) methods, which have allowed sources to cover photon energies from the tens to hundreds of $\mathrm{eV}$. The second method is the X-ray free-electron laser (XFEL). ${ }^{3}$ A free-electron laser is created by the interaction of photons and electrons that travel at near light speed through an undulator, or sequence of undulators, where magnets are arranged in an alternating pattern. As the electron bunch proceeds through the undulators, an increasing number of photons are emitted. Then, if the photon trajectories can be maintained through the undulator, they will repeatedly interact with the near light speed electron bunch, causing micro-bunching of the electrons and coherent emission of more photons. This leads to the so-called self-amplified spontaneous emission (SASE) effect. ${ }^{4,5}$ The first lasing of an FEL in the hard X-ray regime $(>1 \mathrm{keV})$ was achieved in 2009 at the LCLS by SLAC (USA), ${ }^{6}$ followed by SACLA (RIKEN, Japan; 2011), ${ }^{7}$ PAL-XFEL (PAL, Korea; 2016), ${ }^{8}$ the European XFEL (Germany; 2016), ${ }^{9}$ and SwissFEL (PSI, Switzerland; 2017).$^{10}$ Since the first generation of SASE hard X-ray photons ten years ago, an amazing number of new results have been produced using XFELs. On this occasion, we are happy to publish this themed issue on the application of XFELs to investigations in chemical and material science.

An XFEL is an accelerator-based light source like synchrotron radiation (SR) sources. In a storage ring, the electron bunch circulates around the ring at almost light speed and emits radiation ranging from the microwave to the X-ray regime, depending on the properties of the photon generation source (e.g. bending magnet or undulator). In an XFEL, the near light speed electron bunch is produced by a linac (linear accelerator), before being injected into the undulator chain. After being used to generate photons, the electrons are then dumped, making an XFEL a single-pass accelerator. An XFEL produces markedly more monochromatic X-rays than a storage ring undulator (typically $0.5 \%$ in SASE; $0.01 \%$ in seeded mode). The radiation wavelength, $\lambda$, is given as

$$
\lambda=\lambda_{\mathrm{u}} / 2 \gamma^{2} \cdot\left(1+K^{2} / 2\right)
$$

where $\lambda_{\mathrm{u}}, \gamma, K$ are the undulator period length, the electron beam energy, and the undulator parameter, respectively. Both SR and XFEL light sources provide $\mathrm{X}$-ray pulses, but the pulse durations are different, i.e. the width of a SR pulse is of the order of tens to hundreds of picoseconds $\left(10^{-12} \mathrm{~s}\right)$, while that of an XFEL is in the femtosecond regime $\left(10^{-15} \mathrm{~s}\right)$. Due in part to this decrease in pulse duration of 3 orders of magnitude, the peak brilliance of an XFEL is $10^{31-33}$ photons per ( $\mathrm{s} \mathrm{mm} \mathrm{mrad}^{2} 0.1 \% \mathrm{BW}$ ), while that of SR is $10^{23-25}$ photons per $\left(\mathrm{s} \mathrm{mm} \mathrm{mrad}^{2}\right.$ $0.1 \% \mathrm{BW}$ ). A second important difference in the photon properties is the transverse coherence, which is $100 \%$ for XFELs, whereas most current SR sources have approximately $0.1 \%$. These remarkable properties of XFEL photons have pioneered new frontiers in science and technology. 
An ultra-short X-ray pulse from an XFEL enables us to carry out ultrafast experiments. The pump-probe technique is typically used for the measurement of ultrafast dynamics, where a reaction is initiated with a short optical pulse and the XFEL pulse is then used to probe the system after a variable time delay. XFELs have been used for pump-probe X-ray absorption, diffraction, and imaging with femtosecond temporal resolution, providing researchers with all the advantages these $\mathrm{X}$-ray spectroscopic and scattering tools provide. Ultra-intense X-ray pulses are used for structural biology, mainly through the serial femtosecond crystallography (SFX) method, where diffraction patterns from micro-sized protein crystals are recorded shot-to-shot, enabling X-ray damage-free structures to be measured. Another important application for ultraintense X-rays is for non-linear optical phenomena, such as two-photon absorption, sum frequency generation, saturable absorption, and so on. A final but important feature is the ability to use the spatial beam coherence for techniques such as coherent diffractive imaging (CDI), which is a developing technique used to image nanostructures.

This themed issue collects a series of articles that cover the most recent achievements of XFEL applications to chemistry. All chemical reactions occur with the excitation of electrons, followed by a structural change. X-ray absorption fine structure spectroscopy (XAFS) is suitable for determining the local electronic and geometrical structure around an X-ray absorbing atom. Electronic structure changes can be probed using X-ray absorption near edge structure measurements (XANES). DOI: 10.1039/C9CP01332F, 10.1039/C9CP03019K, $10.1039 / \mathrm{C} 9 \mathrm{CP} 03483 \mathrm{H}$ and 10.1039/ C9CP03677F present ultra-fast XAFS studies on photoabsorption processes. DOI: 10.1039/ C9CP03483H demonstrates femtosecond EXAFS measurements of a solution-phase system, which require a significantly higher $\mathrm{S} / \mathrm{N}$ ratio for the data analysis than XANES techniques. DOI: 10.1039/ C9CP03677F is a challenging work studying surface adsorbates, which inherently have much smaller concentrations than bulk samples. Absorption and emission spectroscopy give more detailed electronic structure information. DOI: 10.1039/C9CP03374B presents analysis of resonant inelastic X-ray scattering (RIXS) data in order to understand the carrier dynamics of a water splitting photocatalyst. In addition to results from X-ray spectroscopy, three experimental papers using other X-ray methods are included in this issue. DOI: 10.1039/C9CP03658J uses coherent diffraction imaging at an XFEL. The second is DOI: $10.1039 / \mathrm{C} 9 \mathrm{CP} 03638 \mathrm{E}$, which explores the ultrafast decomposition reaction and ionization of diiodomethane molecules. The last paper (DOI: 10.1039/C9CP03951A) discusses attosecond pulses from an XFEL and their application to attosecond transient absorption spectroscopy. As experimental development requires complementary theoretical development for the correct interpretation of results, four theoretical papers, DOI: 10.1039/ C9CP03019K, 10.1039/C9CP03688A, 10.1039/ C9CP03695D, 10.1039/C9CP03696B are also included in this issue, emphasizing the importance of new theoretical tools to understand the results measured using these new methods.

Most current XFELs use the SASE method to generate X-ray pulses. A SASE XFEL has a different energy spectrum from pulse to pulse. If the X-ray pulse is monochromatized, this photon energy instability results in large intensity variations in the pulses. An analogy would be that a SASE XFEL is like a wild rodeo horse, while a seeded XFEL is like a tamed horse under control of the rider. Consequently, a seeded XFEL exhibits improved properties in the longitudinal phase space, which is particularly important for non-linear X-ray science. An important development in the field of surface science is X-ray sum frequency generation (SFG), which has a high sensitivity for surfaces and interfaces. X-ray SFG provides information on the structure and electronic state at the atomic level in the given reaction environment and can help elucidate the chemical processes that occur on surfaces with high time resolution.

In summary, with the advent of SASE XFELs in the past decade, we have embarked on the road to new science. In the next decade, we will see the flourishing of XFEL science using seeded XFEL and/or other new XFEL sources.
We hope this themed issue will be a guide for new XFEL applications in chemistry and material science.

\section{References}

1 H. H. Telle, A. G. Ureņa and R. J. Donovan, Laser Chemistry: Spectroscopy, Dynamics and Applications, John Wiley \& Sons, 2007.

2 D. L. Andrews and A. A. Demidov, An Introduction to Laser Spectroscopy, Springer Science \& Business Media, 2012.

3 U. Bergman, V. K. Yachandra and J. Yano, X-Ray Free Electron Lasers: Applications in Materials, Chemistry and Biology, Royal Society of Chemistry, 2017.

4 K.-J. Kim, Z. Huang and R. Lindberg, Synchrotron Radiation and Free-Electron Lasers, Cambridge University Press, 2017.

5 K.-J. Kim, Phys. Rev. Lett., 1986, 57, 1871-1874.

6 P. Emma, R. Akre, J. Arthur, R. Bionta, C. Bostedt, J. Bozek, A. Brachmann, P. Bucksbaum, R. Coffee, F.-J. Decker, Y. Ding, D. Dowell, S. Edstrom, A. Fisher, J. Frisch, S. Gilevich, J. Hastings, G. Hays, P. Hering, Z. Huang, R. Iverson, H. Loos, M. Messerschmidt, A. Miahnahri, S. Moeller, H. D. Nuhn, G. Pile, D. Ratner, J. Rzepiela, D. Schultz, T. Smith, P. Stefan, H. Tompkins, J. Turner, J. Welch, W. White, J. Wu, G. Yocky and J. Galayda, Nat. Photonics, 2010, 4, 641.

7 T. Ishikawa, H. Aoyagi, T. Asaka, Y. Asano, N. Azumi, T. Bizen, H. Ego, K. Fukami, T. Fukui, Y. Furukawa, S. Goto, H. Hanaki, T. Hara, T. Hasegawa, T. Hatsui, A. Higashiya, T. Hirono, N. Hosoda, M. Ishii, T. Inagaki, Y. Inubushi, T. Itoga, Y. Joti, M. Kago, T. Kameshima, H. Kimura, Y. Kirihara, A. Kiyomichi, T. Kobayashi, C. Kondo, T. Kudo, H. Maesaka, X. M. Maréchal, T. Masuda, S. Matsubara, T. Matsumoto, T. Matsushita, S. Matsui, M. Nagasono, N. Nariyama, H. Ohashi, T. Ohata, T. Ohshima, S. Ono, Y. Otake, C. Saji, T. Sakurai, T. Sato, K. Sawada, T. Seike, K. Shirasawa, T. Sugimoto, S. Suzuki, S. Takahashi, H. Takebe, K. Takeshita, 
K. Tamasaku, H. Tanaka, R. Tanaka,

T. Tanaka, T. Togashi, K. Togawa,

A. Tokuhisa, H. Tomizawa, K. Tono,

S. Wu, M. Yabashi, M. Yamaga,

A. Yamashita, K. Yanagida, C. Zhang,

T. Shintake, H. Kitamura and

N. Kumagai, Nat. Photonics, 2012, 6, 540.

8 S. Ko, H.-S. Kang, H. Heo, C. Kim, G. Kim, C.-K. Min, H. Yang, S. Y. Baek, H.-J. Choi, G. Mun, B. R. Park, Y. J. Suh, D. C. Shin, J. Hu, J. Hong, S. Jung, S.-H. Kim, K. Kim, D. Na, S. S. Park, Y. J. Park, Y. G. Jung, S. H. Jeong, H. G. Lee and S. Lee, Appl. Sci., 2017, 7, 479.

9 T. Tschentscher, C. Bressler, J. Grünert, A. Madsen, A. Mancuso, M. Meyer, A. Scherz, H. Sinn and U. Zastrau, Appl. Sci., 2017, 7, 592.
10 C. J. Milne, T. Schietinger, M. Aiba, A. Alarcon, J. Alex, A. Anghel,V. Arsov, C. Beard, P. Beaud, S. Bettoni, M. Bopp, H. Brands, M. Brönnimann, I. Brunnenkant, M. Calvi, A. Citterio, P. Craievich, M. Csatari Divall, M. Dällenbach, M. D’Amico, A. Dax, Y. Deng, A. Dietrich, R. Dinapoli, E. Divall, S. Dordevic, S. Ebner, C. Erny, H. Fitze, U. Flechsig, R. Follath, F. Frei, F. Gärtner, R. Ganter, T. Garvey, Z. Geng, I. Gorgisyan, C. Gough, A. Hauff, C. P. Hauri, N. Hiller, T. Humar, S. Hunziker, G. Ingold, R. Ischebeck, M. Janousch, P. Juranić, M. Jurcevic, M. Kaiser, B. Kalantari, R. Kalt, B. Keil, C. Kittel, G. Knopp, W. Koprek, H. T. Lemke, T. Lippuner, D. Llorente Sancho, F. Löhl, C. Lopez-Cuenca,
F. Märki, F. Marcellini, G. Marinkovic, I. Martiel, R. Menzel, A. Mozzanica, K. Nass, G. L. Orlandi, C. Ozkan Loch, E. Panepucci, M. Paraliev, B. Patterson, B. Pedrini, M. Pedrozzi, P. Pollet, C. Pradervand, E. Prat, P. Radi, J.-Y. Raguin, S. Redford, J. Rehanek, J. Réhault, S. Reiche, M. Ringele, J. Rittmann, L. Rivkin, A. Romann, M. Ruat, C. Ruder, L. Sala, L. Schebacher, T. Schilcher, V. Schlott, T. Schmidt, B. Schmitt, X. Shi, M. Stadler, L. Stingelin, W. Sturzenegger, J. Szlachetko, D. Thattil, D. M. Treyer, A. Trisorio, W. Tron, S. Vetter, C. Vicario, D. Voulot, M. Wang, T. Zamofing, C. Zellweger, R. Zennaro, E. Zimoch, R. Abela, L. Patthey and H.-H. Braun, Appl. Sci., 2017, 7, 720. 